\title{
Avaliação Física e Operacional dos Terminais Rodoviários de Passageiros com base no MITERP e NBR 9050 - Estudo de Caso Troncal Sul de Mato Grosso
}

\section{Physical and Operational Evaluation of Passenger Bus Terminals based on MITERP and NBR 9050 - Case Study Southern Troncal of Mato Grosso}

\author{
${ }^{1}$ Frank Lennox Oliveira Queiroz e ${ }^{2}$ Sergio Luiz Moraes Magalhães \\ 1 Engenheiro Civil- Faculdade de Arquitetura Engenharia e Tecnologia FAET- UFMT, Mestrando pela \\ Universidade Federal do Rio de Janeiro \\ Programa de engenharia de transportes - Coppe (frankqueiroz16@gmail.com) \\ 2
}

Recebido em: Fevereiro 2016 Aceito em: Maio 2016 Publicado em: junho 2016

\begin{abstract}
Resumo: O transporte rodoviário é o mais importante do Brasil no que concerne ao transporte de passageiros. Sua capilaridade faz com que os pontos mais longínquos deste país sejam atingidos. Devido a sua importância e crescente demanda, é necessário verificar se os serviços e a infraestrutura estão de acordo com as normas vigentes. Este trabalho objetiva analisar as condições operacionais e físicas dos terminais rodoviários do troncal sul de Mato Grosso. Para isso, a metodologia de pesquisa se baseou na pesquisa de campo, para descrever aspectos fundamentais a um terminal, e a pesquisa bibliográfica. Os resultados obtidos ressaltaram uma necessidade de adequação dos terminais principalmente no que toca à acessibilidade, item que pesou muito na má avaliação das edificações. Percebeu-se, também, a necessidade de estudos voltados à atualização do MITERP, bem como estudos específicos ao projeto arquitetônico e acessibilidade nos terminais rodoviários de Mato Grosso
\end{abstract}

Palavras- Chave: Terminais rodoviários; avaliação; MITERP.

\begin{abstract}
Road transport is the most important in Brazil with regard to passenger transport. Its capillarity makes the most distant points of the country are met. Because of its importance and growing demand, it must ensure that services and infrastructure are in line with current regulations. This work aims to analyze the operational and physical condition of the bus terminals in southern Mato Grosso trunk. For this, the research methodology was based on field research, to describe key aspects to a terminal, and literature. The results highlighted a need to adapt the terminals especially when it comes to accessibility, item weighed heavily on poor assessment of buildings. It was noticed, too, the need for studies aimed at updating the MITERP as well as specific studies to architectural design and accessibility in bus terminals of Mato Grosso.
\end{abstract}

Keywords: Bus terminals, evaluation, MITERP.

\section{INTRODUÇÃO}

O deslocamento é uma atividade imprescindível à sociedade atual. Ainda que o avanço da tecnologia proporcione a possibilidade de cumprir funções através do mundo virtual, o deslocamento é, e sempre será, uma atividade essencial, sobretudo, quando se trata de relações familiares ou de lazer. Tamanha importância transforma o setor de transporte de passageiros em um grande destaque ao crescimento econômico e desenvolvimento social.

Neste cenário, no Brasil, o transporte de passageiros por meio do modal rodoviário por ônibus tem predominância. No ano de 2013, cerca de 140 milhões de passageiros foram transportados considerando-se viagens interestaduais e internacionais (ANTT, 2015 apud SUPAS/GERPA, 2013). A importância do deste modal para o país é evidenciada quando se compara com o modal aéreo. No mesmo ano o transporte aéreo atendeu a demanda de 109 milhões de passageiros, considerando viagens de passageiros, considerando viagens dentro do território nacional e internacionais (ANAC, 2013). 
Tal relevância se deve à tamanha capilaridade do transporte por ônibus, que chega aos destinos mais remotos e isolados do país. Segundo a ABRATI (2015), o ônibus chega a mais de 5500 municípios do Brasil, enquanto o transporte aéreo só consegue atender a 120 cidades. Nenhum outro modal é tão eficiente no transporte de passageiros quanto $\mathrm{o}$ modal rodoviário para distância de em torno de $400 \mathrm{~km}$. É um meio de transporte seguro, flexível, pontual e relativamente barato.

Para atender com qualidade a demanda de passageiros deste vasto país, o transporte rodoviário de passageiros por ônibus tem uma estrutura mínima muito bem definida. Tal estrutura, conforme Costa (2010), deve ser composta por no mínimo: terminais, veículos e vias.

No que concerne aos terminais dentro deste sistema, eles desempenham o importante papel de integrar o passageiro à cidade destino, bem como propiciar acessibilidade às diversas localidades por meio de um planejamento de tráfego eficiente. Assim, o terminal rodoviário de

passageiros proporciona desenvolvimento urbano se planejado conforme as recomendações criadas para tais edificações.

Entretanto, nem todos os terminais rodoviários foram criadas em concordância com a norma que rege a criação dos mesmos. Dessa maneira, surgem inúmeros prédios que em nada se assemelham em terminais, haja vista a falta de infraestrutura mínima para cumprir as funções fundamentais de um TRP (Terminal Rodoviário de Passageiros).

Nos subitens seguintes é feita a revisão da literatura, enfatizando os pontos que serão analisados neste estudo.

\section{Pontos de acesso}

Para se estudar terminais rodoviários, primeiramente, faz-se necessário diferenciá-los dos pontos de parada. Tal distinção é essencial na escolha das cidades visitadas.

Tanto pontos de parada quanto terminais são considerados pontos de acesso. Os pontos de parada estão localizados nos passeios públicos, onde é realizado o embarque e o desembarque de passageiros de ônibus e bondes. No geral, o controle de acesso ao sistema de transporte, isto é, a bilhetagem, é feita no interior dos veículos.

Já os modos de transporte como o metrô, trem urbano e ônibus de viagens de longa distância, realizam o embarque e desembarque em estruturas denominadas terminais. O controle de acesso, neste caso, é feito fora dos veículos, na entrada do terminal.

Manual de Implantação de Terminais Rodoviários de Passageiros (MITERP)

O MITERP é um manual constituído por onze partes que abrangem todas as fases da vida útil de um terminal rodoviário. São descritas recomendações quanto à implantação, bem como a aspectos relativos à

classificação e dimensionamento, localização, projeto arquitetônico, programação visual, atividades comerciais, entre outros.

Nos próximos capítulos desta revisão é feita a explanação dos itens recomendados pelo MITERP $\mathrm{e}$ analisados neste estudo.

\section{Classificação dos terminais rodoviários de passageiros}

Um terminal rodoviário de passageiro pode ser classificado de várias maneiras.

A classificação mais conveniente para este estudo é a do MITERP que usa como base o número médio de partidas diárias. Por meio deste parâmetro fixam-se padrões uniformes de dimensionamento para atender às situações características de demanda. 
A tabela 1 evidencia como é feita a classificação de um terminal. A média das partidas é o parâmetro principal, ou seja, aquele que define a classe TRP, todos os outros são dependentes do primeiro.

Tabela 1 - Classificação dos terminais rodoviários de passageiros

\begin{tabular}{|c|c|c|c|}
\hline Classes & $\begin{array}{l}\text { Número Médio de } \\
\text { Partidas Diárias }\end{array}$ & $\begin{array}{c}\text { Número de Plataformas } \\
\text { de Embarque }\end{array}$ & $\begin{array}{c}\text { Número de Plataformas } \\
\text { de Desembarque }\end{array}$ \\
\hline $\mathbf{A}$ & 1250 a 901 & 62 a45 & 21 a15 \\
\hline B & 900 a 601 & 45 a30 & 15 a10 \\
\hline C & 600 a 401 & 30 a20 & 10 a 7 \\
\hline D & 400 a 251 & 20 a13 & 7 a5 \\
\hline $\mathbf{E}$ & 250 a 151 & 13 a 8 & 5 a3 \\
\hline $\mathbf{F}$ & 150 a 81 & 8 a5 & 3 a2 \\
\hline G & 80 a 25 & 5 a2 & 2 a1 \\
\hline $\mathbf{H}$ & 24 a 15 & 1 & 1 \\
\hline
\end{tabular}

\section{Dimensionamento}

O MITERP (1986) estabelece normas de dimensionamento em sua segunda parte. Primeiramente, é feita a classificação tendo como base a demanda. Posteriormente, é feito o dimensionamento por meio de tabelas. Estas tabelas fornecem itens como: área do salão de espera, número de lavatórios e chuveiros, número de assentos, número de bebedouros, entre outros pontos.

As exigências diferem de classe pra classe, sendo maior na classe A e menor na classe $\mathrm{H}$, inclusive alguns setores deixam de ser obrigatórios quando a classificação do terminal se aproxima de $\mathrm{H}$.

\section{Localização do TRP}

Um dos aspectos mais relevantes para um terminal eficaz é a sua localização. Neste ponto é realizada a análise de uma série de elementos que fazem com que a chegada ao TRP seja mais fácil ou mais difícil.

Para Gouvêa (1980), a localização deve principalmente estar aliada à estrutura do sistema de transporte e de acordo com as características de desenvolvimento da malha urbana. Deve ser feita de modo que o terminal não venha a se tornar obsoleto, mas, ao contrário, possa atrair um maior número de usuários e proporcionar maior eficiência ao sistema de transporte.

Para as empresas transportadoras, as condições de acesso dos ônibus ao sistema rodoviário é o ponto mais importante.

Entende-se que a proximidade aos corredores de transporte é imprescindível à atração destas empresas, pois influi no tempo de percurso dos ônibus dentro da área urbana.

Além do acesso, é importante que a edificação esteja localizada em um ponto no qual seja possível a expansão do terminal, de maneira a minimizar os efeitos de sua inevitável obsolescência.

\section{Programação Visual}

Segundo MITERP (1986), a programação visual tem dois objetivos, são eles:

proporcionar um sistema de informação eficiente que referencie as diversas áreas de um TRP e;

estabelecer um padrão de sinais que não promova poluição visual.

Programação visual é composta principalmente pelas placas que indicam a localização de instalações em um TRP. Na Figura 01 está representada a 
simbologia do setor comercial de um terminal.

Figura 1 - Exemplo de programação visual

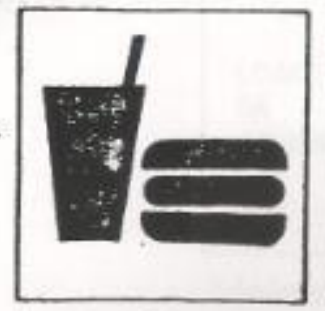

Lanchonete

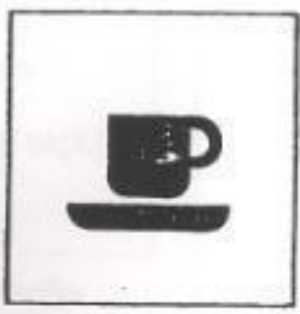

Café

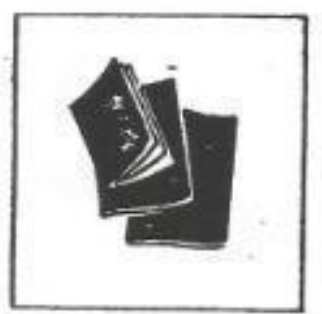

Jornais

e Revistas

Fonte: MITERP (1986)

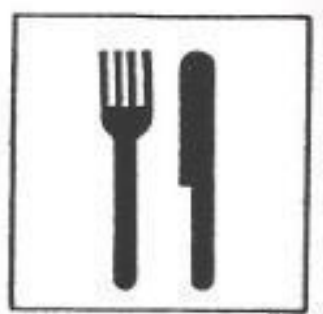

Restaurante

\section{Operação}

Entende-se por operação em um terminal, a gama de ações que são desenvolvidas em um TRP, a fim de tornar possível e eficiente o embarque e desembarque de passageiros.

Neste trabalho os tópicos analisados serão: guarda-volumes, posto de informações, sistema de sonorização, sistema de vídeo para avisos, divulgação de horários de partidas e chegadas e rede de relógios.

\section{Acessibilidade}

A possibilidade de um indivíduo portador de deficiência ter acesso às diversas áreas de uma determinada edificação é denominada acessibilidade. demasiadamente sucinta é adequada ao objetivo deste estudo.

A análise da acessibilidade dos terminais é feita com base com base na NBR 9050 e Cartilha da Acessibilidade da ANTT. Os pontos analisados foram: pisos, rampas, vagas para estacionamento, telefone público, balcões e sanitários.

\section{MATERIAIS E MÉTODOS}

Tendo em vista a extensão do estado de Mato Grosso, optou-se pela escolha de uma região a fim de melhorar a qualidade das análises. $\mathrm{O}$ troncal Sul, composto pelas BR-163 e BR-364, é a região de estudo deste trabalho (Figura 2).
Figura 2 - Troncal Sul de Mato Grosso (BR 163/364)

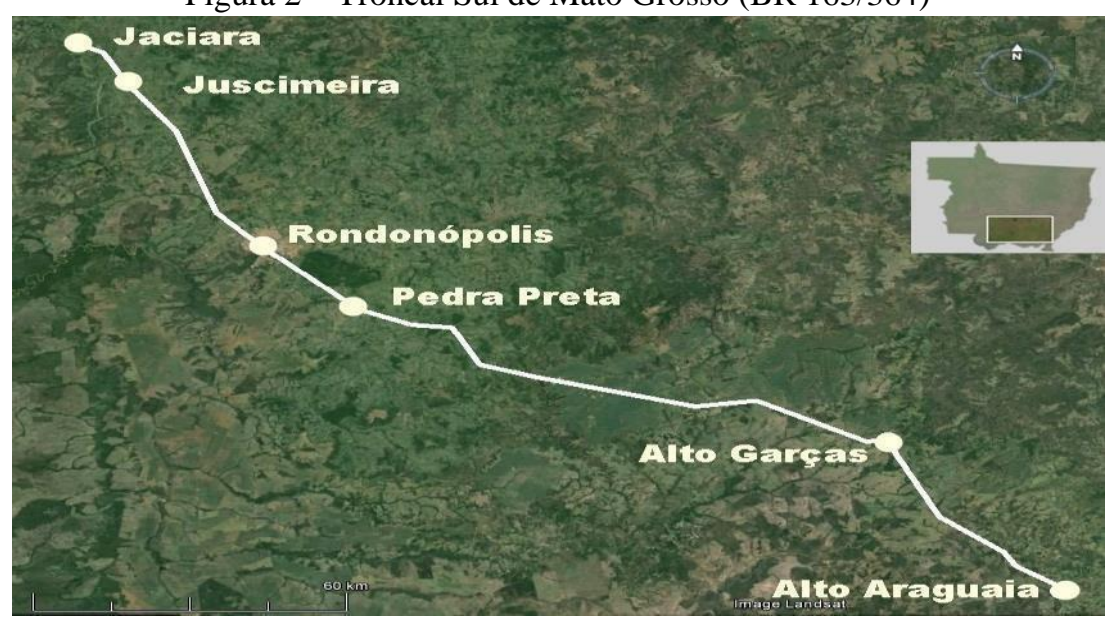

Fonte: Google Maps (2016) 
Após escolher a região foi necessário apontar quais cidades seriam estudadas. Os municípios que possuem terminal rodoviário de passageiros são: Jaciara, Juscimeira, Rondonópolis Pedra Preta, Alto Garças e Alto Araguaia.

\section{Ambiente de pesquisa}

Ambiente de pesquisa é aquele espaço no qual há a possibilidade de observação, descrição e análise. Quando o objeto de estudo é terminal rodoviário existe uma gama de tópicos a serem observados, o estudo deles acarreta na completa caracterização de um TRP.

Pode-se dividir o ambiente de pesquisa em um terminal em espaço externo e espaço interno. O espaço externo compreende as vias do entorno e os polos geradores de tráfego que compõem a localidade.

Quanto ao espaço interno entendem-se os setores nos quais há o fornecimento de produtos e serviços em um terminal. Bilheterias, sanitários, bares e restaurantes são exemplos de localidades analisadas neste estudo.

\section{Coleta de dados de campo}

Para análise do espaço externo utilizou-se um GPS (Global Position System). Tal aparelho arquiva às coordenadas da localidade, o que torna possível o posterior estudo do entorno do terminal.

Quando se limita a avaliação aos espaços internos, ainda pode-se dividir em duas partes: atividades comerciais e serviços de operação. Quanto às atividades comerciais deve ser realizada a quantificação dos bares, restaurantes, lojas e conveniências, entre outras áreas similares existentes no terminal.

A coleta dos dados de serviços de operação consiste na quantificação e descrição dos estacionamentos, bilheterias e sanitários.

\section{Pesquisa bibliográfica}

A pesquisa bibliográfica realizada para este trabalho de graduação fez uso dissertações, artigos, trabalhos de graduação e cartilhas de órgãos nacionais que discorrem sobre terminais rodoviários, bem como sobre acessibilidade em prédios públicos.

Para fazer o dimensionamento dos terminais rodoviários, fez-se necessário o material da AGER/MT com o número de embarques e desembarques nos terminais de todo o estado.

\section{RESULTADOS}

A análise de dados obtidos será feita tomando-se como base o MITERP para os itens: localização, dimensionamento, operação, programação visual e atividades comerciais. Como o Manual ainda não contempla recomendações à acessibilidade seguiu-se a NBR 9050 que estabelece critérios referentes a este ponto.

\section{Pontos analisados}

Cada item citado foi subdivido conforme o disposto nas tabelas de 2 a

7. Após a análise de cada item, calculou-se uma nota para cada terminal, com base na porcentagem de atendimento às recomendações.

Tabela 2 - itens para análise da localização

\begin{tabular}{c} 
Localização \\
\hline Acesso ao terminal \\
Tempo de percurso \\
Possibilidade de expansão \\
\hline
\end{tabular}

Para a avaliação da localização foram levados em conta parâmetros como facilidade de acesso, tráfego das vias do entorno, tempo de percurso dentro da cidade, possibilidade de expansão do terminal. 
Tabela 3 - itens para análise do dimensionamento

\begin{tabular}{|c|}
\hline Dimensionamento \\
\hline Área total das plataformas \\
\hline Área de espera \\
\hline Estacionamento \\
Ponto de taxi \\
Telefone público \\
Assentos \\
Bebedouro \\
Lavatórios \\
Vaso Sanitário \\
Chuveiro \\
Mictório \\
\hline
\end{tabular}

O dimensionamento depende substancialmente da classificação do terminal.

Tabela 4 - itens para análise da operação

\section{Operação}

\begin{tabular}{|c|}
\hline Guarda-volumes \\
\hline Posto de informações \\
Sistema de sonorização \\
Sistema de vídeo \\
Divulgação de horários de \\
partidas e chegadas \\
Rede de relógios \\
\hline
\end{tabular}

Os itens de operação promovem à eficiência no embarque e desembarque no terminal, bem como propiciam conforto e comodidade ao passageiro.

Tabela 5 - itens para análise da programação

\begin{tabular}{c} 
visual \\
\hline Programação Visual \\
\hline Setor de operações \\
Setor de uso público \\
Serviços públicos \\
Setor de administração \\
Setor comercial \\
Empresas transportadoras \\
\hline
\end{tabular}

A programação visual tem o objetivo de proporcionar informações referentes aos diversos locais, instalações e serviços dentro de um terminal, configurando-se como um elemento importante no que toca a eficiência do deslocamento no TRP.

Tabela 6 - itens para análise da atividade comercial

\begin{tabular}{c}
\hline Atividades comerciais \\
\hline Lanchonete \\
Banca \\
\hline
\end{tabular}

Com base na classe do terminal, o MITERP estabelece serviços comerciais necessários.

Para os terminais deste estudo são exigidos, no mínimo, uma lanchonete e uma banca.

Tabela 7 - itens para análise da acessibilidade

\begin{tabular}{c} 
Acessibilidade \\
\hline Rampas \\
Corrimãos \\
Lavatórios acessíveis \\
Bacias Sanitárias \\
acessíveis \\
Pisos antiderrapantes \\
Vagas para indivíduos de \\
mobilidade reduzida \\
Sinalização horizontal \\
Sinalização vertical \\
Sinalização tátil \\
Piso regular \\
Telefones públicos \\
acessíveis \\
Balcões e bilheterias \\
Assentos exclusivos \\
\hline
\end{tabular}

Os itens desta tabela são essenciais à análise da acessibilidade dos terminais considerados.

\section{Classificação dos terminais}

A classificação de um terminal rodoviário é feita através da quantificação de embarques que ocorrem no local.

Conforme o número de partidas consulta-se a Tabela 1 e de imediato obtém-se a classe do terminal rodoviário. $\mathrm{O}$ número de partidas foi obtido com o banco de dados da AGER. 
Queiroz et.al.,Avaliação Física e Operacional dos Terminais Rodoviários de Passageiros com base no MITERP e NBR 9050 Estudo de Caso Troncal Sul de Mato Grosso.E\&S - Engineering and Science, (2016), 5:1.

A classificação é a seguinte:

Jaciara: classe $\mathrm{G}$

Juscimeira: classe $\mathrm{G}$

Rondonópolis: classe $\mathrm{F}$

Pedra Preta: classe $\mathrm{H}$

Alto Garças: classe $\mathrm{G}$

Alto Araguaia: classe G

Tabela 8 - Porcentagem de atendimento às normas

\begin{tabular}{ccccccc}
\hline & Localização & Dimension. & Operação & $\begin{array}{c}\text { Prog. } \\
\text { Visual }\end{array}$ & $\begin{array}{c}\text { Ativ. } \\
\text { Comerc. }\end{array}$ & Acessib. \\
\hline Jaciara & $100 \%$ & $78 \%$ & $17 \%$ & $17 \%$ & $100 \%$ & $31 \%$ \\
Juscimeira & $67 \%$ & $10 \%$ & $0 \%$ & $17 \%$ & $50 \%$ & $15 \%$ \\
Rondonópolis & $100 \%$ & $80 \%$ & $50 \%$ & $83 \%$ & $100 \%$ & $69 \%$ \\
Pedra Preta & $0 \%$ & $82 \%$ & $0 \%$ & $33 \%$ & $50 \%$ & $23 \%$ \\
Alto Garças & $67 \%$ & $50 \%$ & $0 \%$ & $17 \%$ & $50 \%$ & $0 \%$ \\
$\begin{array}{c}\text { Alto } \\
\text { Araguaia }\end{array}$ & $33 \%$ & $55 \%$ & $0 \%$ & $50 \%$ & $50 \%$ & $0 \%$ \\
\hline
\end{tabular}

Na Tabela 8 estão dispostos os resultados obtidos da análise física e operacional dos terminais rodoviários estudados.

Por meio das porcentagens obtidas, calculou-se a média aritmética. Por meio da média calcularam-se as notas finais para cada localidade.

As notas foram as seguintes:

Jaciara: 5,7

Juscimeira: 2,6

Rondonópolis: 7,9

Pedra Preta: 3,1

Alto Garças: 3,1

Alto Araguaia: 3,1

\section{DISCUSSÃO}

As avaliações no que se refere à localização foram satisfatórias na maioria das cidades estudadas. Entretanto, os terminais de Pedra Preta e Alto Araguaia foram mal avaliados devido à distância do troncal, assim como, no caso da segunda cidade, o aclive acentuado para se chegar ao terminal. TRPs como o de Juscimeira e Alto Garças não obtiveram notas máximas devido à impossibilidade de expansão da edificação.
Quanto ao dimensionamento, o baixo número de assentos na área de espera e a ausência de bebedouros foram os problemas mais recorrentes. Os terminais que receberam as piores notas neste tópico também tiveram problemas quanto à área coberta mínima exigida aos ônibus. O desempenho do TRP de Juscimeira é notável, haja vista que somente $10 \%$ dos itens analisados foram atendidos.

Quanto à operação, todos os terminais tiveram avaliação catastrófica. Destaca-se apenas Rondonópolis que atingiu $50 \%$ de adequações às normas. Serviços como sistemas de vídeo para avisos e divulgação de horários de partidas e chegadas não foram observados em nenhum dos terminais analisados.

A programação visual também não teve resultados positivos na maioria dos terminais do troncal sul. Apenas Rondonópolis e Alto Araguaia, embora com problemas, conseguiram alcançar um bom desempenho neste parâmetro. As demais cidades possuem somente a identificação das empresas transportadoras em seus TRPs.

As atividades comerciais exigidas para as classes dos terminais deste estudo são: lanchonete e banca de jornal. Em todas as edificações há ao menos uma lanchonete, entretanto somente em Rondonópolis e Jaciara há bancas ou similares.

Os resultados mais preocupantes são os de acessibilidade, pois nenhum dos terminais alcançou $70 \%$ de adequação. Entretanto, o índice mais inquietante é dos terminais de Alto Garças e Alto Araguaia que não apresentaram nenhum dos itens considerados nesta pesquisa.

Os elementos que mais estiveram presente nos terminais foram as rampas, entretanto, cabe salientar que tais 
rampas, na maioria das edificações não possuem corrimãos. Os balcões foram avaliados como adequados, nas cidades que pontuaram, tendo em vista que atendem a NBR 9050. Quanto à acessibilidade, o destaque positivo é novamente o terminal de Rondonópolis, visto que apresenta grande parte dos itens analisados, restando apenas

adequações nos estacionamentos (sinalização horizontal e vertical) e a sinalização tátil para deficientes visuais.

\section{CONCLUSÃO}

O terminal de Jaciara tem localização privilegiada, entretanto a operação e programação visual são os aspectos que demandam séria readequação.

$O$ terminal de Juscimeira foi avaliado como o pior da região sul (BR 163-364) de Mato Grosso. Este terminal necessita, com urgência, de adaptações para promover acessibilidade $\mathrm{e}$ comodidade, por meio do correto dimensionamento e operação.

O terminal de Rondonópolis apresentou a melhor avaliação deste estudo, em todos os tópicos atendeu ao menos $50 \%$ das exigências. Todavia, os equipamentos de operação do terminal devem ser melhorados. Também, alguns locais devem ser adaptados para ficar de acordo com as normas de acessibilidade.

Em Pedra Preta a localização teve avaliação nula, isto se deve a distância do terminal ao troncal. Recomenda-se um estudo de viabilidade para a instalação de um terminal às margens da estrada, com intuito de tornar a edificação menos ociosa. Além da localização, aspectos relacionados à operação e acessibilidade também devem ser reparados ou inseridos.

No terminal de Alto Garças se destaca a avaliação nula no que concerne à acessibilidade. Recomenda- se a urgente adequação do local aos itens mínimos citados em normas.

No terminal de Alto Araguaia, além da péssima avaliação em operação e acessibilidade, nota-se um desempenho igualmente ruim no que toca a localização. Assim como em Pedra Preta, recomenda-se um estudo que demonstre a viabilidade de se instalar um terminal mais próximo da rodovia.

No terminal de Alto Garças se destaca a avaliação nula no que concerne à acessibilidade. Recomendase a urgente adequação do local aos itens mínimos citados em normas.

No terminal de Alto Araguaia, além da péssima avaliação em operação e acessibilidade, nota-se um desempenho igualmente ruim no que toca a localização. Assim como em Pedra Preta, recomenda-se um estudo que demonstre a viabilidade de se instalar um terminal mais próximo da rodovia.

\section{AGRADECIMENTOS}

Agradecimento especial aos técnicos da AGER/MT que realizaram a primeira coleta de dados de campo. Tal coleta foi imprescindível à análise do item dimensionamento.

\section{REFERÊNCIAS}

ABNT. Associação Brasileira de Normas Técnicas. NBR 9050: Acessibilidade a edificações, mobiliário, espaços e equipamentos urbanos. Rio de Janeiro, 2004.

\begin{tabular}{llll} 
ABRATI & - & \multicolumn{2}{c}{ ASSOCIAÇÃO } \\
BRASILEIRA & DAS & EMPRESAS & DE \\
TRANSPORTE & TERRESTRE & DE \\
PASSAGEIROS. & Revista & $N^{\circ}$ & 82. \\
Brasília-DF. 2015. & & &
\end{tabular}


Queiroz et.al.,Avaliação Física e Operacional dos Terminais Rodoviários de Passageiros com base no MITERP e NBR 9050 Estudo de Caso Troncal Sul de Mato Grosso.E\&S - Engineering and Science, (2016), 5:1.

AGÊNCIA NACIONAL DE COSTA, M. de F. H. Uso de Modelos AVIAÇÃO CIVIL. Anuário do de Localização Para Diagnóstico de transporte aéreo de 2013.

AGÊNCIA NACIONAL DE TRANSPORTES TERRESTRES.

Cartilha da Acessibilidade, 2009. Rede de Terminais de Transporte de Passageiros - Estudo de Caso em Terminais Rodoviários do Estado do Ceará. Universidade Federal do Ceará. Ceará, 2010

AGENCIA NACIONAL DE TRANSPORTES TERRESTRES.

Apresentação. Disponível em: <http://www.antt.gov.br/index. $\mathrm{php} /$ content/view/4890/Apresentacao.ht ml>. Acesso em: 20 de abril de 2015

AGER - Agência de Regulação dos Serviços Públicos delegados do estado de Mato Grosso. Planilha para dimensionamento dos terminais de Mato Grosso. Mato Grosso. 2014.

GOUVÊA, V. B. Contribuição ao Estudo de Implantação de Terminais Urbanos de Passageiros. Instituto Militar de Engenharia. Rio de Janeiro. 1980.

DNER. Departamento Nacional de Estradas e Rodagens (1986). MITERP Manual de Implantação de Terminais Rodoviários de Passageiros. 